\title{
Noninvasive Brain-Computer Interface Driven Hand Orthosis
}

\author{
Christine E. King, Po T. Wang, Masato Mizuta, David J. Reinkensmeyer, An H. Do, \\ Shunji Moromugi, and Zoran Nenadic
}

\begin{abstract}
Neurological conditions, such as stroke, can leave the affected individual with hand motor impairment despite intensive treatments. Novel technologies, such as brain-computer interface (BCI), may be able to restore or augment impaired motor behaviors by engaging relevant cortical areas. Here, we developed and tested an electroencephalogram (EEG) based BCI system for control of hand orthosis. An able-bodied subject performed contralateral hand grasping to achieve continuous online control of the hand orthosis, suggesting that the integration of a noninvasive BCI with a hand orthosis is feasible. The adoption of this technology to stroke survivors may provide a novel neurorehabilitation therapy for hand motor impairment in this population.
\end{abstract}

\section{INTRODUCTION}

Many stroke survivors are affected by hand motor impairment, and despite best therapy, functional recovery typically plateaus 3-6 months after stroke [1]. In addition, treatments for individuals affected by distal upper extremity weakness are rather limited, and so alternative biomechanical therapies have been sought. Brain-computer interface (BCI) may offer a novel approach to restoring or augmenting lost motor behaviors in stroke patients. In addition to controlling an external device to restore these motor functions, BCIs may facilitate the reinforcement of spared connections between the post-stroke motor cortex and spinal motor pools relevant to the lost motor function [2], [3], [4].

Generally, BCIs control an external device by analyzing neurophysiological signals associated with a motor process and translating them into commands to drive the device in real time. Successful BCI applications include the control of computer cursors [5], virtual keyboards [6], movement within virtual reality environments [7], robotic arms [8], functional electrical stimulation devices [9], and hand orthoses for stroke rehabilitation [10], [11]. However, previous BCI-hand orthosis systems either required extensive training [10], or lacked continuous online control [11].

This work was supported in part by the Roman Reed Spinal Cord Injury Research Fund of California under Grant RR10-281.

C.E. King, P.T. Wang, and Z. Nenadic are with the Department of Biomedical Engineering, University of California, Irvine, CA 92697, USA kingce@uci.edu, ptwang@uci.edu, znenadic@uci.edu

M. Mizuta and S. Moromugi are with the Department of Mechanical Systems Engineering, Nagasaki University, Nagasaki, Japan mizuta.masato@gmail.com, smoromug@nagasaki-u.ac.jp

D.J. Reinkensmeyer is with the Department of Mechanical and Aerospace Engineering, University of California, Irvine, CA 92697, USA dreinken@uci.edu

A.H. Do is with the Department of Physical Medicine and Rehabilitation, University of California, Irvine, CA 92697, USA and@uci.edu

\section{METHODS}

\section{A. Overview}

An electroencephalogram (EEG) based BCI system was integrated with a hand orthosis [12] suitable for rehabilitation of impaired hand motor function. To demonstrate purposeful control, the proposed system (Fig. 1) was tested on an able-bodied subject engaged in a contralateral control handgrasping paradigm. More specifically, the subject underwent alternations of voluntary right hand idling and repetitive grasping while her EEG patterns were decoded in real time and used to trigger the corresponding action of the left hand orthosis. This paradigm was chosen since in able-bodied subjects, ipsilateral control paradigm would produce confounding results, as it would be difficult to resolve voluntary and BCI-mediated orthosis movements.

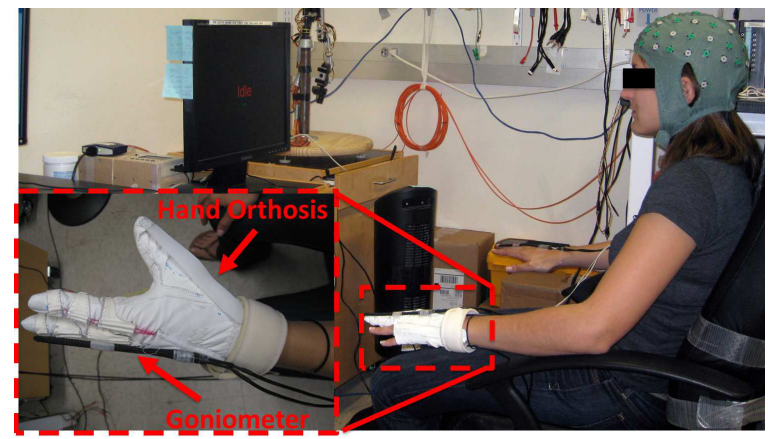

Fig. 1. EEG underlying right hand idling and grasping is measured by an EEG cap and sent to a computer for analysis. The computer then sends control commands to an orthotic glove [12] mounted on the left hand. Two electrogoniometers [13] mounted on each hand measure hand movements. The subject follows textual cues displayed on a computer screen.

\section{B. Signal Acquisition}

An able-bodied female (age 24, with $\sim 10 \mathrm{hr}$ of BCI experience) was seated $\sim 1 \mathrm{~m}$ from a computer screen that displayed textual cues (Fig. 1). EEG was recorded using a 63-channel cap (Medi Factory, Heerlen, The Netherlands) with $\mathrm{Ag}-\mathrm{AgCl}$ electrodes arranged according to the 10-20 International Standard. Conductive gel was applied to all electrodes and the electrode impedances were maintained at $<10 \mathrm{k} \Omega$ by abrading the scalp with a blunt needle. The EEG signals were amplified, band-pass filtered (4-40 Hz), digitized (sampling rate: $256 \mathrm{~Hz}$, resolution: 22 bits), and acquired in a common average reference mode using two linked 32-channel EEG amplifier systems (MindMedia, RoermondHerten, The Netherlands). Finally, the EEG signals were streamed in real time and analyzed using custom-written MATLAB (MathWorks, Natick, MA) programs. 


\section{Training Procedure \& Offline Signal Analysis}

The subject underwent a 20 -min training session consisting of 200 trials of alternating epochs $(6 \mathrm{sec})$ of idling and repetitive right hand grasping while her EEG was recorded. To identify grasping and idling epochs in EEG, a custom-made electrogoniometer [13] was mounted on the metacarpophalangeal joint of the right middle finger. The goniometer signals were acquired with an auxiliary data acquisition system (Biopac Systems, Goleta, CA), and were synchronized with EEG signals using a common pulse train.

The training EEG data were then analyzed offline. First, channels with muscle artifacts were automatically detected by our signal processing algorithm and excluded from further analysis. The continuous 20-min EEG record was then split into 100 idle and 100 grasping trials based on the goniometer signals. For each trial, the 6-30 $\mathrm{Hz}$ EEG power spectrum was calculated in $2-\mathrm{Hz}$ bins. This resulted in a $12 \times C$ dimensional data matrix, where $C$ is the number of retained EEG channels. To facilitate subsequent classification, lowdimensional features were extracted from the input data using a combination of classwise principal component analysis [14] and approximate information discriminant analysis [15]. This yielded a nonlinear transformation (mapping) from the $12 \times$ $C$ data space into a one-dimensional (1D) feature space. Subsequently, these 1D spatio-spectral features, $f^{\star}$, were inputted into a Bayesian classifier:

$$
\text { Class }=\left\{\begin{array}{l}
\text { Idle, if } P\left(I \mid f^{\star}\right)>P\left(G \mid f^{\star}\right) \\
\text { Grasp, otherwise }
\end{array}\right.
$$

where $P\left(I \mid f^{\star}\right)$ and $P\left(G \mid f^{\star}\right)$ are the posterior probabilities of idling and grasping classes given the observed feature, $f^{\star}$, respectively. The classification accuracy of the Bayesian classifier 1 was then assessed by performing 5 runs of stratified 10-fold cross-validation (CV) [16]. The parameters of the feature extraction mapping and the classifier, referred to as the prediction model, were saved for real-time EEG classification during online evaluation.

\section{Online Signal Analysis \& BCI-Hand Orthosis Evaluation}

For online operation, $0.75 \mathrm{sec}$ overlapping EEG segments were acquired in real time with a refresh rate of $2 \mathrm{~Hz}$. These segments were then processed using the same method described in Section II-C. The posterior probabilities of idle and grasping classes were then calculated based on these salient EEG features and were used to control the grasping and extension of the hand orthosis. Prior to obtaining control of the orthosis, a short calibration procedure was performed to determine the posterior probability thresholds such that false state transitions during hand orthosis operation are minimized. To accomplish this, the BCI system was set to run in the online mode while the orthosis was turned off. The subject was then prompted to alternate between $\sim 20$-sec epochs of idling and repetitive hand grasping for a total of $3 \mathrm{~min}$. Using the prediction model developed in Section II-C, real-time EEG signal analysis was performed, and the posterior probabilities of grasping and idling given data were calculated every $0.5 \mathrm{sec}$. Fig. 2 shows the empirical distributions of these probabilities. Using the median (50\% quartile), two thresholds were chosen based on these histograms: one to trigger the transition from grasping to idle state and another to trigger the transition from idle to grasping state. This choice of threshold was found to yield a good compromise between false alarms and omission errors. Finally, to facilitate smooth online BCI operation, these probabilities were averaged over a $1.5 \mathrm{sec}$ period, and the average posterior probabilities were compared to these thresholds in order to control the hand orthosis.

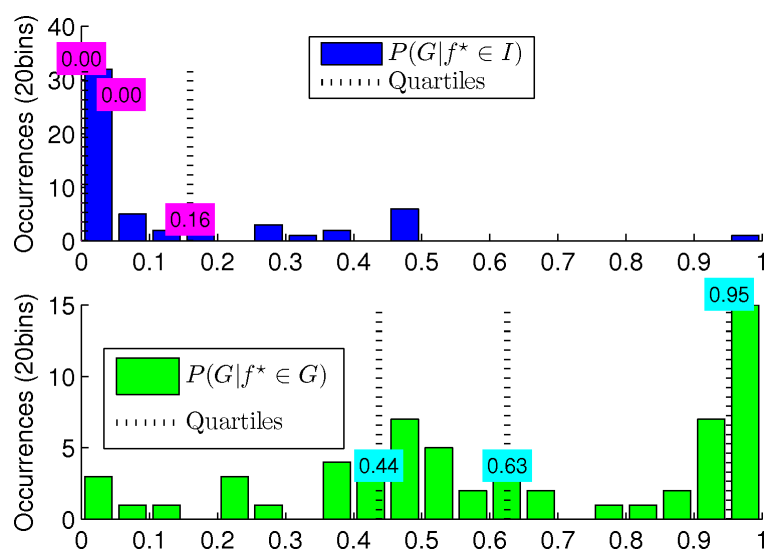

Fig. 2. (Top) Histogram of the posterior probability of grasping (G) given that the underlying action is idling (I). (Bottom) Histogram of the posterior probability of grasping $(\mathrm{G})$ given that the underlying action is grasping. The dashed lines mark the $25 \%, 50 \%$, and $75 \%$ quartiles.

To evaluate the performance of the BCI-hand orthosis system, the subject was instructed via textual cues to perform 10 alternating 10-sec epochs of right hand idling and repetitive grasping to induce BCI-mediated orthosis grasping of the left hand. Then, readings from two electrogoniometers were used to compare voluntary right hand grasping and BCIhand orthosis mediated left hand grasping. The goniometer traces were smoothed using a 100-msec Gaussian window and thresholds were set manually to determine grasping and idling epochs. A time series, $x$, describing voluntary right hand movements was then defined as:

$$
x[i]= \begin{cases}0, & \text { if } \quad i \in I \\ 1, & \text { if } \quad i \in G\end{cases}
$$

where $i=1,2 \cdots, N$, and $N$ is the number of samples in the goniometer trace. A time series, $y$, describing BCI-hand orthosis mediated grasping, was defined in a similar manner. The normalized cross-covariance function between the time series $x$ and $y$ was then calculated as:

$$
\rho(m)=\frac{\sum_{i=1}^{N}(x[i+m]-\bar{x})(y[i]-\bar{y})}{\sqrt{\sum_{i=1}^{N}(x[i]-\bar{x})^{2}} \sqrt{\sum_{i=1}^{N}(y[i]-\bar{y})^{2}}}
$$

where $m \in[-N+1, N-1]$ is the lag between the sequences $x$ and $y$, and $\bar{x}$ and $\bar{y}$ are the sample means of the two sequences, respectively. The latency between voluntary and BCI-hand orthosis mediated left hand grasping was 
then found as the lag with maximal cross-covariance, i.e. $m^{\star}=\arg \max _{m} \rho(m)$. Subsequently, the temporal correlation between $x$ and $y$ was found to be: $\rho^{\star}=\rho\left(m^{\star}\right)$. In addition to these measures, the performance was quantified by calculating the number of false alarms and omissions. An omission was defined as the absence of a BCI-hand orthosis mediated grasping epoch within the duration of any voluntary hand grasping epoch. Similarly, the initiation of a BCI-hand orthosis mediated hand-grasping epoch within any idling epoch was considered a false alarm.

\section{RESULTS AND DISCUSSION}

\section{A. Offline Performances}

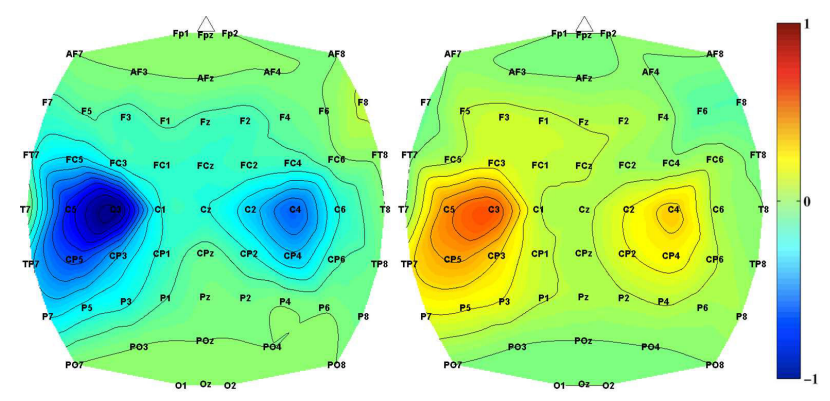

Fig. 3. Spatio-spectral feature extraction maps at high $\beta$-band $(18-20 \mathrm{~Hz})$. Values close to +1 and -1 indicate brain areas of importance for classifying EEG data into idling and grasping classes. Since feature extraction mapping is piecewise linear, there are two maps; one adapted to idling class (left) and one adapted to grasping class (right).

By performing 10-fold $\mathrm{CV}$, an offline classification accuracy of $95.3 \% \pm 0.6 \%\left(\mathrm{p}<3.0866 \times 10^{-25}\right)$ was achieved. Analysis of the prediction model showed that the EEG power changes in the $\beta$-band observed over the electrode C3 were the most informative features for classification (Fig. 3). This electrode likely corresponds to the right hand motor representation area, located on the lateral left primary motor cortex. This finding is consistent with the anatomy of motor homunculus, and similar spatial locations and spectral features were implicated in related studies [9], [11].

\section{B. Online Performances}

To assess the feasibility of integrating BCI and orthosis systems, its function was evaluated online, where the subject alternated between 10 ten-sec epochs of right hand idling and repetitive grasping. In response to hand grasping epochs, a BCI-mediated orthosis grasping and extension were typically induced (Fig. 4). Performance was quantified by calculating the temporal correlation between the right hand movement epochs and the corresponding BCI-mediated orthosis movement epochs, the average lag between these epochs, and the number of false alarms and omissions.

After a total of 5 online sessions, the average lag between the epochs of voluntary grasping/idling and BCI-hand orthosis grasping/idling was $2.24 \pm 0.19$ sec. The temporal correlation between these epochs calculated at each online session's corresponding lag value averaged to $0.778 \pm 0.06$ (Table I). Also, the average number of false alarms and omissions was determined to be $0.4 \pm 0.89$ and $1.8 \pm 1.10$,

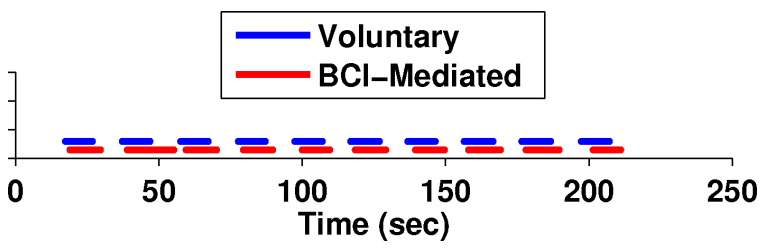

Fig. 4. Blue traces mark the 10 epochs of repetitive right hand grasping, whereas gaps indicate idling epochs. Red traces mark the epochs of BCImediated orthosis grasping of the left hand. Both traces were estimated as described in Section II-D.

respectively. Finally, the best online performance (Session 5) resulted in no false alarms or omissions and a lag time of $2.5 \mathrm{sec}$ with a temporal correlation of 0.84 .

TABLE I

PERFORMANCES OVER THE 5 ONLINE SESSIONS. THE ASTERISK CORRESPONDS TO THE ONLINE SESSION WITH THE BEST PERFORMANCE (SHOWN IN FIG. 4).

\begin{tabular}{ccccc}
\hline Session & Lag $(\mathrm{sec})$ & $\rho$ & OM & FA \\
\hline 1 & 2.1 & 0.69 & 2 & 2 \\
2 & 2.0 & 0.79 & 0 & 2 \\
3 & 2.3 & 0.80 & 0 & 2 \\
4 & 2.3 & 0.77 & 0 & 3 \\
$5^{*}$ & 2.5 & 0.84 & 0 & 0 \\
\hline Average & $2.24 \pm 0.20$ & $0.78 \pm 0.06$ & $0.40 \pm 0.89$ & $1.8 \pm 1.10$ \\
\hline
\end{tabular}

Since stroke patients with hand motor impairments are unable to execute full grasping movement, we tested the performance of the above system by utilizing repetitive micromovements (simultaneous $5^{\circ}-10^{\circ}$ flexion of all fingers). This experiment was also implemented using the contralateral control paradigm and was performed with the same able-bodied subject. Analysis of a single online session (Fig. 5) yielded the lag of $1.889 \mathrm{sec}$ between the epochs of right hand micromovement/idling and BCI-hand orthosis grasping/idling. In addition, the temporal correlation between these epochs calculated at this lag value was 0.772 . Finally, note that this session had no false alarms or omissions.

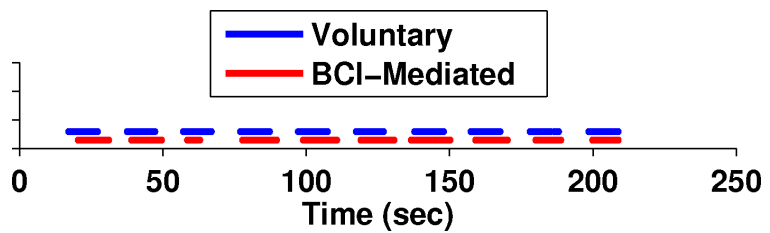

Fig. 5. The same as in Fig. 4, except that subject performed repetitive micromovements of the right hand.

\section{Discussion}

These results demonstrate that continuous BCI-mediated orthosis grasping/idling could be reliably controlled using a contralateral hand-grasping paradigm. The high offline classification accuracy (>95\%) translated into the subject being able to achieve continuous online control of the interface. Moreover, only a single training session (20 min) was required to develop a reliable prediction model for feature extraction and classification of EEG signals. Besides cross-validation, the validity of this model was confirmed by the feature extraction mapping (Fig. 3), which is consistent with the anatomical organization of the motor cortex. This result is even more impressive given that the design of the 
prediction model is entirely data driven without imposing any anatomical constraints. Finally, the model was validated online, first through a calibration procedure, which showed a clear separation of posterior probabilities (Fig. 2), followed by real-time online control (Figs. 4 and 5). In particular, the high correlation between the offline performance and the real-time online performances indicates that no overfitting occurred in the design of the prediction model.

Due to inability to resolve voluntary and BCI-mediated orthosis movements in able-bodied individuals, the present study used contralateral control paradigm. To apply this technology to the neurorehabilitation of stroke survivors with hand weakness, an ipsilateral control paradigm should be used. Through the use of attempted or partially executed movements of the affected hand, followed by the activation of the orthosis, post-stroke individuals may be able to restore hand grasping behavior in an intuitive manner and use this system as an automated physiotherapeutic rehabilitation tool.

In addition to an ipsilateral control paradigm, the adoption of this technology to neurorehabilitation applications will most likely require the elimination of the latency between the onset of voluntary or attempted movement/idling and BCI-hand orthosis mediated movement/idling. The observed latency is partly caused by the averaging of the posterior probability over a $1.5 \mathrm{sec}$ period (see Section II-D) during online operation. Also, there is a natural delay in the eventrelated desynchronization/resynchronization of EEG sensorimotor rhythms [17] which are concomitant with the execution/cessation of movements, respectively. For example, the peak desynchronization was found to occur $\sim 1$ sec after initiation [17] or imagination [18] of movements, which may be responsible for the delay in the BCI-hand orthosis system response. A potential solution to this problem is to reduce the posterior probability averaging window (albeit at the expense of increase the false alarm and omission rates) or to use our data-driven algorithm to search for relevant EEG features in the time domain, such as readiness potentials, which may be observed as early as $1 \mathrm{sec}$ before initiation of a self-paced motor behavior [19]. However, further research is required to implement these solutions, as both changes in the training paradigm and signal-processing methodology will need to be implemented such that the novel EEG features associated with movement intention can be reliably detected.

Finally, the post-stroke brain is expected have undergone cortical reorganization that may result in EEG signal features that deviate from classical features underlying hand-grasping behavior. The data-driven feature extraction method demonstrated here can find optimal combinations of EEG signal features directly associated with hand-grasping behavior in the post-stroke cortex. This method may facilitate reliable BCI operation of the orthosis and in turn, help reinforce connections between the post-stroke brain areas underlying hand grasping and the corresponding spinal motor pools.

\section{CONCLUSIONS}

This study demonstrates that the integration of a noninvasive EEG-based BCI system with a hand orthosis is feasible. With minimal training, an able-bodied subject was able to use contralateral control paradigm to continuously operate BCI-hand orthosis in real time. Adoption of this technology to neurorehabilitation of stroke survivors with hand motor impairments will require further development including testing of the system's function under ipsilateral control paradigm and minimization of the latency between movement intentions and hand-orthosis response.

\section{REFERENCES}

[1] S.-M. Lai, S. Studenski, P.W. Duncan, and S. Perera. Persisting consequences of stroke measured by the stroke impact scale. Stroke, 33(7):1840-1844, 2002.

[2] P.M. Rossini, C. Altamura, F. Ferreri, J-M. Melgari, F. Tecchio, M. Tombini, P. Pasqualetti, and F. Vernieri. Neuroimaging experimental studies on brain plasticity in recovery from stroke. Eura Medicophys, 43(2):241-254, 2007.

[3] D. Broetz, C. Braun, C. Weber, S.R. Soekadar, A. Caria, and N. Birbaumer. Combination of brain-computer interface training and goaldirected physical therapy in chronic stroke: a case report. Neurorehabil Neural Repair, 24(7):674-679, 2010.

[4] J.J Daly, R. Cheng, J. Rogers, K. Litinas, K. Hrovat, and M. Dohring. Feasibility of a new application of noninvasive brain computer interface (BCI): a case study of training for recovery of volitional motor control after stroke. J Neurol Phys Ther, 33(4):203-211, 2009.

[5] J.R Wolpaw and D.J. McFarland. Control of a two-dimensional movement signal by a noninvasive brain-computer interface in humans. Proc Natl Acad Sci U S A, 101(51):17849-17854, 2004.

[6] N. Birbaumer, N. Ghanayim, T. Hinterberger, I. Iversen, B. Kotchoubey, A. Kübler, J. Perelmouter, E. Taub, and H. Flor. A spelling device for the paralysed. Nature, 398(6725):297-298, 1999.

[7] R. Leeb, D. Friedman, G.R. Müller-Putz, R. Scherer, M. Slater, and G. Pfurtscheller. Self-paced (asynchronous) BCI control of a wheelchair in virtual environments: A case study with a tetraplegic. Comput Intell Neurosci, page 79642, 2007.

[8] D.M. Taylor, S.I. Tillery, and A.B. Schwartz. Direct cortical control of 3D neuroprosthetic devices. Science, 296(5574):1829-1832, 2002.

[9] G. Pfurtscheller, G.R. Müller, J. Pfurtscheller, H.J. Gerner, and R. Rupp. 'thought'-control of functional electrical stimulation to restore hand grasp in a patient with tetraplegia. Neurosci Lett, 351(1):33-36, 2003.

[10] E. Buch, C. Weber, L.G. Cohen, C. Braun, M.A. Dimyan, T. Ard, J. Mellinger, A. Caria, S. Soekadar, A. Fourkas, and N. Birbaumer. Think to move: a neuromagnetic brain-computer interface (BCI) system for chronic stroke. Stroke, 39(3):910-917, 2008.

[11] C.W. Chen, C.C.K. Lin, and M.S. Ju. Hand orthosis controlled using brain-computer interface. J. Med. Biol. Eng, 29:234-241, 2006.

[12] S. Moromugi, K. Kawakami, K. Nakamura, T. Sakamoto, and T. Ishimatsu. A tendon-driven glove to restore finger function for disabled. In Proc. ICCAS-SICE, pages 794-797, 2009.

[13] P.T. Wang, C.E. King, A.H. Do, and Z. Nenadic. A durable, lowcost electrogoniometer for dynamic measurement of joint trajectories. Medical Engineering \& Physics, 33(5):546-552, 2011.

[14] K. Das and Z. Nenadic. An efficient discriminant-based solution for small sample size problem. Pattern Recogn, 42(5):857-866, 2009.

[15] K. Das and Z. Nenadic. Approximate information discriminant analysis: A computationally simple heteroscedastic feature extraction technique. Pattern Recogn, 41(5):1548-1557, 2008.

[16] R. Kohavi. A study of cross-validation and bootstrap for accuracy estimation and model selection. In Int. Joint C. Art. Int., pages 1137$1145,1995$.

[17] G. Pfurtscheller and A. Aranibar. Evaluation of event-related desynchronization ERD preceding and following voluntary self-paced movement. Electroencephalogr Clin Neurophysiol, 46(2):138-146, 1979.

[18] G. Pfurtscheller, C. Neuper, D. Flotzinger, and M. Pregenzer. EEGbased discrimination between imagination of right and left hand movement. Electroencephalogr Clin Neurophysiol, 103(6):642-651, 1997.

[19] B. Libet, C.A. Gleason, E.W. Wright, and D.K. Pearl. Time of conscious intention to act in relation to onset of cerebral activity (readiness-potential). The unconscious initiation of a freely voluntary act. Brain, 106 (Pt 3):623-642, 1983. 